\title{
APRECIACIONES SOBRE EL SHOCK EN OBSTETRICIA
}

\author{
POR LUIS ANGULO RODRÍGUEZ
}

Insuficiencia periférica aguda, Colapso circulatorio periférico, Asistolia periférica, shock.

Todas estas denominaciones tiene el síndrome de insuficiencia circulatoria, cuando el corazón no es la causa primitiva del proceso y en el que el mecanismo básico, es la desproporción entre el volumen de sangre circulante y el diámetro del lecho vascular.

Las escuelas norteamericanas hacen una división, en relación con la intensidad y forma de aparición, y así llaman colapso o shock primario a la insuficiencia circulatoria periférica repentina y de corta duración, y shock verdadero o shock secundario a la insuficiencia circulatoria periférica prolongada, asignándole a parece una división más que todo didáctica, ya que los términos colapso y shock son indivisibles en su concepto, toda vez que no son sino insuficiencia circulatoria periférica esencialmente, siendo hasta el presente un sindrome complejo e incompletamente comprendido y estudiado clínicamente.

En lo que al "shock obstétrico" respecta, no parece sea necesario hacer de él una entidad independiente, de etiología y fisiopatogenia propias, como semeja darlo a entender su denominación, pues parece evidente que en él se conjugan, en la generalidad de los casos, diversos factores desencadenantes y favorecedores, que son los que caracterizan los que se ha dado en llamar específicamente shock hemorrágico, shock traumático, quirúrgico, anestésico, etc.; en estos el factor etiológico es distinto tanto como su fisiopatogenia, siendo agrupados bajo el nombre genérico de shock por la resultante final que es el colapso vascular periférico.

Y si para la aparición de un shock obstétrico, concurren factores, apreciable cada uno de ellos en diferente intensidad, la predominancia de uno de ellos sería en realidad la desencadenante 
y obrarian los demás como favorecedores de tal manera que con esa predominancia llegaría a caracterizar al shock obstétrico con su etiología y fisiopatogenia. De lo anteriormente expuesto es lógico deducir que para denominar más apropiada y científicamente e' shock obstétrico, debe agregarse la causa o factor más notorio y asi tendríamos las expresiones, shock hemorrágico obstétrico, shock traumático obstétrico, etc.

Sintomatología. Cuadro clinico.

Generales: estado de somnolencia, con sensación de abatimiento que en ocasiones se convierte en agitación y angustia; sensibilidad disminuída como también el sensorio, conservándose cierto grado de conciencia. Hiperpnea, respiración rápida y generalmente superficial. Náuseas y vómito; mucosas secas, evidenciable en las conjuntivas y en especial en los labios y boca y que se manifiesta por sed imperiosa; malestar epigástrico.

Cutáneos: Palidez, que en ocasiones se combina con cianosis, notoria especialmente está última en los labios, lóbulo de la oreja y lechos ungüeales, piel fría y húmeda o sudorosa, con temperatura cutánea disminuída.

Circulatorios: Taquicardia y taquisfigmia, convirtiéndose en muchas ocasiones en pulso filiforme y aún llegando a desaparecer o mejor a hacerse imperceptible. Hipotensión sistólica y diastólica, en especial sistólica por coexistir vasoconstricción; la capacidad funcional del corazón está potencialmente intacta, sin lesión ciel miocardio y sin lesión o mal funcionamiento valvular; ruidos cardiacos débiles; venas periféricas virtualmente colapsadas y exangües.

\section{Fisiopatogenia.}

Seguiremos en rasgos generales la de Antonio Battro, por parecernos la más apropiada.

a) Disminución del volumen de sangre circulante, hipovolenia, que es el principal factor en la determinación del shock y cuyas causas pueden ser: primero, disminución real del volumen total de sangre, con lo que se hace insuficiente para llenar en forma adecuada el lecho vascular, como en casos de pérdida de sangre por hemorragia o por paso de una gran parte de líquido a. los tejidos al aumentar la permeabilidad de los capilares (plas- 
modiálisis), o por pérdida de ese líquido en vómitos y trasudación.

En el caso de pérdida de sangre por hemorragias, no necesariamente debe existir relación con el volumen de sangre perdida, sino que puede ser la resultante en la falta de respuesta de los automatismos de compensación circulatoria; por lo cual, no siendo indispensable que exista estricta relación directa entre cantidad de sangre perdida y presentación e intensidad del shock, con hemorragias de discretas proporciones pueden existir sintomas de colapso grave. Razones estas que tiene en cuenta el Obstetra y evita todo sistema para acelerar tanto el trabajo como el parto y el alumbramiento, de no exigirlo así circunstancias especiales y precisas, y porque conoce las incidencias de hernorragia uterina después de maniobras como la de Kristeller y Credé, particularmente si se efectúan en forma desmedida o brusca, casos todos en los que toma las medidas convenientes para prevenir o anticiparse a la aparición de los síntomas del shock. Y ya, viéndose abocado a la presencia del estado de shock durante la gestación, acuden a su imaginación cuadros que le facilitan el diagnóstico de entidades como la ruptura de un embarazo estópico, la apoplejía utero-placentaria, la ruptura uterina, etc.

El otro mecanismo de colapso por hipovolemia, originado por la pérdida de líquidos, ora internamente por aflujo anormal de ellos a los tejidos, o ya externamente por la deshidratación intensa y prolongada, por vómitos y trasudación tiene su explicación en el desequilibrio físico-químico de la sangre y en la imposibilidad orgánica de reaccionar apropiadamente, circunstancias en las cuales se formará un círculo vicioso que se puede expresar así:

Plasmodiálisis

Vasodilatación periférica

Hipovolemia

Asfixia de los centros vasomotores

En pacientes con grandes pérdidas de líquidos se ve alterado €l equilibrio hidrosalino por pérdida de electrolitos en especial los iones sodio y cloro y una notoria hemoconcentración; la fuga de estos elementos hace que el organismo no pueda retener los líquidos suministrados, eliminándolos rápidamente o pasando a los tejidos por anormalidad en la permeabilidad capilar, por lo que no corrigiéndose convenientemente la hipovolemia, se agrava el cuadro con oxigenación deficiente de los tejidos, lo que traerá 
consigo la hiperventilación con pérdida de anhídrido carbónico y ciisminución en la excitación de los centros cardio-respiratorios $\mathrm{y}$ reguladores del tono vascular.

Estos mecanismos pueden presentarse durante el trabajo de parto y más frecuentemente en el puerperio inmediato con aparición de shock, máxime si se agregan en estos periodos, factores neurogénicos y psicogénicos que los reforzarán.

En Clínica Obstétrica es conocida la vasodilatación y gran diaforesis y en ocasiones el vómito, que acompañan al parto en su período de trabajo franco y con mayor evidencia hacia el fina! de él, al que se agregan circunstancias que aumentan las características de intensidad, como distocias cervicales, empleo de ocitócicos, presentaciones distócicas, etc.; pérdidas de líquidos que deben ser reparadas ojalá en exceso, y así evitar el desequilibrio hídrico y humoral.

En la ruptura prematura o precoz de las membranas, se piensa en las complicaciones que origina este accidente, procidensias, infección de la cavidad amniótica, mayor duración del trabajo y aumento de las intervenciones en el parto y alumbramiento; pero no hay que olvidar la pérdida de líquido que en esta entidad se efectúa ya que el amnios seguirá secretando, si es que no existe una excitación de su función originada por el vaciamiento repentino de su cavidad y por cierto grado de amniotitis existente a pesar de las medidas de asepsia y antisepsia que se tornen; por eso debe ser (en Obstetricia) otro factor que hay que considerar en la deshidratación que pueda llegar a producir el mecanismo de shock por hemoconcentración.

b) Vasodilatación periférica.- Vasoparálisis, que afectan en especial los pequeños vasos capilares periféricos, pues parece demostrado que en los órganos donde pudiera efectuarse el depósito sanguíneo, hígado, bazo, territorio de la pulmonar, no se realiza, según hechos experimentales de Eppinger y Gauter. Trae como consecuencia la disarmonía entre el continente y el contenido, por aumento en la capacidad del continente, produciéndose el acúmulo y estancamiento de la sangre, con disminución del volumen circulante y la consiguiente baja de la presión arterial, disminución del débito cardiaco, alterándose la irrigación de los tejidos en especial de los centro cerebro-bulbares y del propio corazón.

Este mecanismo se produce por acción sobre los centros vasomotores o por acción directa sobre las paredes vasculares, asignándoseles como principales causas las que siguen: 
19 Tóxicos, ya pueden ser de naturaleza exógena, en especial arsenicales, barbitúricos y anestésicos en el tema que nos ocupa, - endógenos por aumento en la producción de substancias del tipo de la histamina o acetilcolina.

20 Vasodilatación refleja, en que juegan preponderante papel el traumatismo, el dolor y los estímulos psicogénicos y neurogénicos en personas de constitución asténica y vagotánica.

No existe acuerdo respecto a la actitud del organismo materno frente a la acción de los tóxicos y en particular de los hipnóticos y anestésicos, pues mientras unos afirman la existencia ce una mayor tolerancia a ella, otros la niegan y por el contrario expresan la presencia de una labilidad manifiestc explicable por el. recargo en la función orgánica que representa el embarazo en sí. Pero dejando a un lado estos puntos de vista, es factible presumir la existencia de acción tóxica de diverso grado de intensidad, cuando se utilizan los barbitúricos con fines hipnoanalgésicos durante el trabajo, o cuando se emplean los anestésicos por inhalación por tiempo prolongado con el objeto de dilatar o demorar el momento del parto.

La vasodilatación refleja originada por el traumatismo, dolor o estímulos psicogénicos derivados principalmente de estos dos factores ,forman parte importante en la aparición del shock en Obstetricia. El traumatismo puede hallarse aún en partos espontáneos, en el parto rápido en que la descompresión brusca de la cavidad uterina y por ende la abdominal, puedan acarrear la tracción del plejo solar por ptosis viscerales o por la misma descompresión en si; otro tal puede suceder en el vaciamiento repentino de un hidramnios, en una aplicación de forceps o una expresión abdominal, agregándose a este último el traumatismo directo sobre las vísceras, el peritoneo y los ligamentos y fascias que forman la arquitectura de sostén uterina.

Con respecto al dolor, tal es su importancia en la génesis del shock, que él aparece en accidentes como el desprendimiento prematuro de la placenta normoinserta, en la ruptura uterina, etc., sin olvidar que también puede hacerlo al efectuarse dilataciones cervicales sin la conveniente anestesia, exploraciones vaginales y después de maniobras dolorosas como las de Kristeller y Credé.

Como caso que demuestra la producción del shock por el dolor, tenemos el de la paciente que sufriendo de Tabes dorsal (*) y hallándose en trabajo de parto del que no podía percibir sensación

(*) Historia número 257 de 1952. Servicio del Profesor Acosta. Instituto de Protección Materna-Infantil. 
alguna, efectuó una ruptura uterina por distocia pélvica-ósea, momento en el que únicamente le llamó la atención la emisión de sangre por los genitales externos; esta paciente a pesar de presentar dos factores importantes como eran hemorragia y traumatismo, no manifestó síntoma de desmejoramiento alguno de su estado general, conservando la presión arterial y el pulso dentro de límites sensiblemente normales, pudiéndose efectuar la intervención quirúrgica durante la cual únicamente se les transfundieron 500 c.c. de plasma, medicación esta suficiente para mantenerla en condiciones favorables durante el acto operatorio.

El ejemplo clásico de la vasoparálisis es el que se presenta durante la raquianestesia, con vasodilatación del territorio anestesiado e hipotonía de los músculos esqueléticos; la relajación vascular es intensa, tanto en la circulación arterial como en la venosa, predominante y evidenciable en el territorio esplácnico y que es regulada o controlada parcialmente con la aplicación previa de vasopresores o analépticos periféricos para así mantenerse a salvo del cuadro de hipotensión acentuada y aún de shock, al no seguir las normas técnicas en la administración de la anestesia espinal.

c) Aumento de la permeabilidad capilar.- Plasmodiálisis. En este mecanismo de acción, son primitivos los trastornos metabólicos, por aumento de la permeabilidad en las paredes capilares, hecho que ha sido demostrado experimentalmente con la administración de extractos de tejidos normales, hígado, músculo, mucosa y por productos de desintegración proteíca, histamina, adenosina, etc.; producida la hiperpermeabilidad celular, tendrá lugar la pérdida de plasma, de prótidos (especialmente seroalbúmina de molécula de menor tamaño) y alteración del equilibrio iónico, con tendencia a igualar las concentraciones intra y extracelulares y que es denominada destrucción de la homeostasia fisiológica de Cannon.

Ante la fuga de los componentes plasmáticos del torrente circulatorio a los tejidos y en especial a los espacios inter e intracelulares, por haberse disminuído la presión oncótica intravascularmente, con la consiguiente desviación de las constantes biológicas, apreciaremos la reducción del volumen sanguíneo y aparición de la hemoconcentración; hecho fisiopatológico de importancia capital en la producción del colapso vascular periférico. Condición desencadenante de él podrán ser estados de gestosis y €clampsia, por estar interferido en ellos el equilibrio humoral, to 
que se ve favorecido durante el trabajo por la producción de substancias tóxicas del tipo de la histamina, pudiendo coexistii lambién estados de hipoproteinemia en las grandes albuminurias, y factores de orden neuroendocrino.

No podríamos dar por terminada esta exposición sobre la fisiopatogenia del shock, sin mencionar la posible existencia de un factor hormonal, el que pudiera ser la causa eficiente en la aparición de las diversas modalidades de aquél, ya que a más de su similitud con el cuadro que se puede observar en la enfermedad de Addison, no sería aventurado afirmar que por un mecanismo de inhibición suprarrenal de origen neuroendocrino hiciese su aparición el colapso vascular periférico, máxime si durante el embarazo, existe como lo afirman algunos investigadores, un hiposuprarrenalismo medular con posible disminución de su hormona ce secreción interna la epinefrina.

Un hecho sobre el que existe acuerdo es el que los esteroides corticales son indispensables para la normalidad en las funciones orgánicas y los que permiten afrontar numerosas situaciones de esfuerzo y agotamiento; y si como es presumible, a esta insuficiencia hormonal originada por el excesivo consumo de ella, exigido por las características del trabajo y el parto a la que se agregará durante el shock la inhibición en la producción del factor estimulante, hormona adrenocórticotropa (ACTH), se llegará a las condiciones similares del cuadro presentado en los otros mecanismos de shock.

En favor de esta teoría se encuentra la acción notable, sobre los casos llamados de shock irreversible, obtenida con la administración endovenosa de soluciones de ACTH, en que se asiste a una recuperación' espectacular y rápida, en organismos que no habían respondido al tratamiento habitual cuidadosamente instituído. Explícase el hecho porque a más de que esta hormona es la directa excitadora en la producción de las corticales, es capaz de producir la retención de agua y cloruro de sodio, aumentar la glucogenesis y ayudar a la regularización rápida de los niveles de nitrógeno y potasio aumentados en esta entidad.

Deliberadamente hemos dejado para el final la consideración del psiquismo como factor directo en la producción del shock, en relación con sus manifestaciones de ansiedad, temor, incertidumbre y grado de apreciación del dolor, capaces de actuar en pacientes en que se dice existir "labilidad nerviosa" y de sus principales órganos vegetativos, para que intervenga el psiquismo en su reacción frente a ellas. No pudiendo medir el grado de per- 
cepción de todas las manifestaciones internas de que es consciente una persona y menos aún del dolor, que iniciándose por la diferente distribución de los receptores cutáneos y viscerales, es lievado hasta el tálamo óptico, órgano de percepción central, que le dará tonalidad afectiva diferente en cada persona según su umbral a la intensidad de un estímulo, el factor psicógeno no debe ser considerado de poca importancia ya que éste nivel reaccional individual y factores de acostumbramiento y educación que están directamente condicionados al tipo costitucional y temperamental, serán los que hagan posible la producción de un estado de insuficiencia circulatoria periférica aguda.

Dentro de los casos de shock en que la génesis más probable en su aparición, es lo que pudiéramos llamar el componente psiconeurótico, deberían incluirse aquellos casos, que mal llamados se catalogan como "shock obstétrico puro", en los que no existe etiología evidente en su aparición, ya que suelen presentarse después de un trabajo y parto de evolución normal, sin intervención obstétrica, ni administración de medicamentos, con alumbramiento normal y sin que la paciente presente lesión orgánica alguna, pero en los que casi con certeza se puede afirmar la presencia de factores de indole emocional, afectiva y dolorosa que nos puedan explicar la génesis del shock.

Este tipo de colapso tiene selectividad, pudiéramos decir, por una clase social: por la clase acomodada o culta formada por personas de percepción más fina, de reacción emocional más intensa a todo aquello que represente dolor, sufrimiento, miedo, etc., y que explica su ausencia o extrañeza en las que forman la clientela hospitalaria. Tal es el caso de la paciente cuya historia transcribimos y por el que se originó este trabajo. Como se podrá apreciar a su lectura, es singular por haberse presentado la entidad denominada shock de repetición, el que no se pudo prevenir en las primeras oportunidades a pesar de las medidas tomadas para ello, tanto durante la gestación, como durante el trabajo y el parto y después de haber sido sometida a un examen cuidadoso y pormenorizado de todo el organismo.

\section{Pronóstisco}

Es grave en términos generales y está directamente relacionado a la oportunidad en la apreciación de sus causas y en la precocidad del tratamiento adecuado. La determinación de la hemoconcentración sería de importancia capital como examen de rutina, ya que por medio de ella no solamente se podrá hacer 
el pronóstico, sino que principalmente será útil para el diagnóstico precoz deduciendo la intensidad de la pérdida líquida.

\section{Tratamiento.}

Preventivo. Terapéutica sedante nerviosa adecuada, psicoterapia encaminada especialmente a educar la percepción del clolor y su reacción frente a él, práctica de la relajación corporal y psíquica, administración de hipnoanalgésicos a dosis apropiadas, que facilitando el trabajo lo hacen más llevadero. Evitar la producción de dolor, en exploraciones vaginales bruscas y en maniobras durante el parto y alumbramiento; abstenerse del uso excesivo o inmoderado de los ocitócicos; vigilar o prevenir la aparición de hemorragias y tratar de evitar las causas que la originan; colocar a la paciente en las mismas condiciones que requiere todo postoperatorio, rodeándola de las comodidades posibles referentes a evitarle por tiempo innecesario la exposición al frío y la demora en la administración de sedantes.

Curativo.- Reposo absoluto en decúbito y con discreto Trendelenburg, aspiración de vómito, calentamiento indirecto del cuerpo por sistemas de irradiación. Administración intravenosa de plasma sanguíneo electivamente, que restablece la volemia, normaliza el poder oncótico reintegrando los líquidos escapados a los tejidos, disminuye la hemoconcentración mejorando con todo esto la tensión arterial y venosa, la función cardíaca con la consiguiente normalización del pulso y la tensión periférica. En lo precoz de la administración del plasma reside en gran parte el éxito del tratamiento impidiendo el avance de la sintomatología y de la descompensación orgánica que son los que llevan al shock al estado de irreversibilidad por el gran alcance sobre áreas de importancia vital como los centros cerebrobulbares y el corazón.

Las soluciones cloruradas y dextrosadas isotónicas, administradas por vía endovenosa, tienen una acción momentánea y fugaz, disminuyen el poder oncótico del plasma con disminución de la atracción de los líquidos tisulares hacia la sangre, potencialmente representa un peligro por aumentar el edema tisular y ser fuente de complicaciones posteriores como el edema agudo del pulmón o la insuficiencia cardíaca congestiva; de mayor utilidad serían las soluciones hipertónicas que atrayendo líquidos tisulares a la circulación, y reponiendo en cierto grado la pérdida de electrolitos, darán tiempo a la aplicación de otros recursos terapéuticos. 
Las transfusiones de sangre total tienen aplicación en los casos de shock por hemorragia y en los que ésta no haya podido ser controlada convenientemente. En realidad se administran todos los elementos perdidos pero no deja de representar inconveniente la demora en su administración y los riesgos inherentes a ella, a lo que se agrega que lo capital es la reposición del plasma ya que el tratamiento de la anemia que generalmente no es muy intensa, se podrá dejar para momento más oportuno.

Oxigenoterapia.- Actúa favorablemente combatiendo la anoxemia, reduciendo la taquicardia y elevando la presión arterial, se asocia con la administración de anhídrido carbónico que ayuda a regular el equilibrio ácido-básico perdido por la ipreventilación pulmonar y es excitante fisiológico de los centros respiratorios y vasomotores.

Analépticos circulatorios.- Los centrales son de poca eficiencia y de acción en muchas ocasiones contraproducente pues, con ellos sólo se provoca estímulo de la función cardíaca ya de por sí acelerada, y no se obtendrá acción sobre el colapso.

Los analépticos periféricos tienen su indicación en esta entidad como complemento de la plasmoterapia, efedrina y epinefrina administradas por vía intramuscular y de preferencia por vía endovenosa ya que su acción es inmediata y no está interferida su absorción como en las otras vías de administración. Con ello se busca la excitación de los vasos periféricos y la descarga cie reservas sanguíneas que el organismo no es capaz de efectuar por su inhibición.

Sedación de la paciente si existieron estados de dolor, angustia o excitación, con dosis apropiadas de barbitúricos o de los similares sintéticos de la morfina como el Demerol, Dolosal, etc.

Finalmente, si a todas las normas terapéuticas empleadas no se obtuviese reacción en el estado de la paciente, se justifica el empleo de soluciones endovenosas de ACTH, en dosis suficiente, la que no dejará secuela nerviosa alguna y no interferirá la restauración normal ulterior. Con este control y limitación en la dosis se evitarán las manifestaciones de excitación motriz, natremia, cloruremia e hidremia, con expresión de "moon face" y oliguria e hipopotasemia; cuadro este del hiperfuncionalismo corticoadrenal que requiere para su aparición la administración de closis excesivas, dándole así a la droga un amplio margen de acción. 


\section{Historia clínica}

La señora N. N., paciente particular del Profesor Ramírez Merchán, presenta la siguiente historia clínica de sus cinco sucesivas gestaciones, cuatro de las cuales terminaron con parto a término y una con aborto de pocos meses.

Sus antecedentes hereditarios, ginecológicos y patológicos generales carecen de importancia. Como dato interesante, afirma lo inestable de su carácter, en especial durante la gestación, en que se torna taciturna, melancólica, y en extremo preocupada por su estado y por la terminación de él.

Primera gestación.- Vigilada periódicamente fue normal. Alllegar al término inicia trabajo el día 21 de agosto de 1944, a las 23 horas, de características normales y con un tiempo total de curación hasta la expulsión del feto, de 13 horas y 15 minutos. No es necesario el uso de ocitócicos y como única medicación durante el trabajo se le aplica una ampolla de Trasentina por vía intramuscular. Tanto el parto como el alumbramiento se efectúan espontáneamente; no hay lesión perineal. El niño respira de manera espontánea y pesa 3.500 gramos.

Inmediatamente después del alumbramiento se presenta discreta hemorragia, la que después de la aplicación de una ampolla de ergotrate y ligero masaje de la matriz queda controlada satisfactoriamente; cuando todo hacía evidente la terminación feliz cle este caso, imprevistamente, palidece la paciente, se siente desvanecida y manifiesta sensación de muerte inminente, el pulso radial se hace imperceptible, el corazćn late apresuradamente y la respiración tórnase superficial y entrecortada, entrando finalmente en un estado de semiinconsciencia. Se inicia inmediatamente el tratamiento con la inyección de productos del tipo Cardiazol, Coramina y Analepsina que son los llevados en el maletín como drogas de urgencia; cuarenta y cinco minutos después de iniciado el shock se logra aplicar por venoclisis, solución de dextrosa al $10 \%$ asociada con pequeños tonicardíazos. Dos horas después, tiempo durante el cual la situación es angustiosa, es posibie la consecución y aplicación de plasma sanguíneo formulado en cantidad de 500 c. c. Solamente se han transfundido 100 c. c. cuanco ya se aprecia un notorio mejoramiento de las funciones circulatoria y respiratoria y a la que sigue pocos instantes después una franca mejoría del estado general de la paciente que se manifiesta por la recuperación total de la conciencia y el deseo de tomar alguna bebida; se le administra una taza de té. 
Segunda gestación.- Atendida en la Clínica de La Magdalena (historia clínica número 2.214 de 1946). Exactamente año y medio después del anterior, llega con embarazo a término y en trabajo. El embarazo se ha desarrollado normalmente, otra vez se halla el factor psíquico manifestado por el temor de que se repita el accidente del parto anterior, que complica con el "temor de morir asfixiada" por la anestesia por inhalación, hasta el punto de exigir se le exima de ser sometida a ella. Tal exigencia es aceptada y también, para descartar factores favorecedores, no se le efectúan exámenes vaginales de control del trabajo, a excepción de uno al finalizar aquel y que indica una dilatación de 8 centímetros, bolsa íntegra, presentación encajada en OIA. Se le administran antiespasmódicos y con intervalos de una hora, 20 centigramos de nembutal en dosis fraccionadas con el objeto de mantenerla en estado de somnolencia y sedación.

Iniciado el trabajo a las 18 horas del día 10 de diciembre, se efectúa el parto espontáneamente a las 2 horas del día 2, o sea ocho horas después de la iniciación del trabajo; fue necesaria la aplicación de dos unidades de Pitocín por vía intramuscular para la expulsión, ya que las contracciones se habían atenuado posiblemente a causa de la medicación sedante. Nace una niña que llora espontáneamente y que pesa 2.800 gramos. El alumbramiento se efectúa espontánea y normalmente. Treinta minutos cespués, y estando ya en su habitación, se presenta el estado de shock. con cuadro similar al del primer parto, agravándose con crisis eméticas que empeoran el estado general. Se aplica cafeína, dextrosa al $10 \%$ en solución salina por venoclisis, pero el cuadro continúa igual; nuevas dosis de cafeína, coramina y percorten y una hora después se inicia la transfusión de plasma sanguíneo, estableciéndose la mejoría y recuperándose totalmente cuatro y media horas después de iniciado el shock.

Tercera gestación.- El transcurso de ella es normal, pese a io cual se recurre a la colaboración de un escectalista en cardiología, el que después de prolijo examen clínico y electrocardiográfico, diagnostica la presencia de un "síndrome de astenia neurocirculatoria”, formula la administración de coramina-efedrina por via oral desde el séptimo mes de embarazo y la aplicación en el transcurso del trabajo, de dextrosa al $10 \%$ por vía endovenosa a la que se deberá mezclar una ampolla de coramina.

Es obvio suponer el estado de alteración física en que se haliará la paciente con el antecedente de los partos anteriores; al llegar a la clínica presenta ya un estado avanzado de trabajo, 
aproximadamente tres o cuatro horas, pues los datos suministrados por e! examen vaginal, indican una dilatación completa, membranas íntegras, vértice en OP en el estrecho inferior, a pesar de lo cual se le administra la venoclisis prescrita por el cardiólogo. Llevada a la sala de partos rechaza nuevamente la enestesia por inhalación y se efectúa inmediatamente el parto espontáneo. Sucesivamente a la salida del feto se aplica faja compresiva abdominal, también por insinuación del cardiólogo, para evitar el factor traumatismo o descompresión brusca abdominal.

Cinco minutos después del parto se efectúa el alumbramiento y como se presentara discreta hemorragia, para evitar este factor como desencadenante del estado de shock, bajo anestesia general por inhalación, se efectúa revisión de la cavidad uterina que demuestra la expulsión total de los anexos, formándose a continuacićn un buen globo de retracción; pocos segundos después nuevamente se presenta el shock, en esta ocasión se tiene ya listo el plasma sanguíneo que sustituirá a la anterior administración de cextrosa, por lo que la recuperación es más rápida y una nueva dosis de 250 c. c. de plasma produce la restitución completa a los 90 minutos de iniciado el shock.

En las gestaciones siguientes cuarta y quinta, desaparece el cuadro de shock; en la cuarta que termina por un aborto espontáneo y completo de pocos meses, trata de repetir la iniciación de la sintomatología del colapso, con el inconveniente de haberse efectuado en su domicilio, pero una terapéutica psicológica oportuna y eficaz hace desaparecer rápidamente los síntomas insiciiosos que manifestaba.

En la quinta gestación que termina con parto espontáneo y a término el 22 de abril de 1954, se tienen precauciones y se establecen normas encaminadas a lograr la sedación psíquica y a anular el estado de ansiedad e hiperemotividad, lo que junto con la prescripción de hipnoanalgésicos, plasma, y hormona suprarrenal con fines profilácticos durante el trabajo y la apropiada conducción de éste para evitar excesivo dolor, traumatismo y demás factores que pudieran desencadenar el estado de shock, transcurre todo dentro de la mayor normalidad y éxito.

\section{CONCLUSIONES}

1ạ El "shock en Obstetricia", mal denominado "shock Obstétrico", no es una entidad de caracteres especiales sino que obedece a las mismas causas que lo originan fuera del estado grávido puerperal. 
2a Para denominarlo convenientemente, se debería especificar la causa de produccićn: traumática, anestésica, hemorrágica, psíquica, etc.

3ạ Cuando su causa es hemorrágica, no se debe apreciar únicamente como consecuencia directa de la cantidad; hay reacciones reflejas vasomotoras originadas por pequeñas hemorragias que no son menos importantes.

$4^{\text {a }}$ La deshidratación juega papel importante; pérdidas líquidas por diaforesis, vómitos y ruptura prematura de las membranas deben ser convenientemente valoradas.

5a Debe considerarse en su real valor la influencia del psiquismo como causa desencadenante y si axistiere labilidad del sistema nervioso, tomar las precauciones conducentes para evitar la acentuación de tal estado.

6. El dolor debe mitigarse porque no solamente existen los modos de hacerlo sin perjuicio de la paciente, sino que es lo que espera ella del médico.

7a Deben evitarse maniobras que produzcan dolor o traumatismo innecesariamente o sistemas encaminados a disminuir la duración del trabajo y el parto, no con miras técnicas sino exclusivamente personales.

$8^{\text {a }}$ El "shock Obstétrico puro", no existe, ya que ninguno de los mecanismos de un parto normal pueden ser causa de shock; posiblemente en estos casos existe un componente psiconeurótico que no se aprecia convenientemente.

9ạ En la precocidad del tratamiento estriban el éxito de él y la benignidad del pronóstico.

10. Los estados irreversibles generalmente se deben a falta de tratamiento oportuno.

11. La plasmoterapia y los analépticos periféricos en primer lugar y la oxigenoterapia, calentamiento, quietud, etc., en segundo término, debe ser el tratamiento de elección.

12. Dentro de la terapéutica obstétrica podría incluirse el empleo de soluciones de ACTH, para el tratamiento de casos de shock grave, o en los que no se obtiene resultado con otros convenientemente establecidos. 


\section{BIBLIOGRAFIA}

1. RAMIREZ MERCHAN R.- "Shock obstétrico". Rev. Med. de la Acad. Nal. de Med. Col. 49 (570-71).

2. BENINCORE C. J.- "El estado de shock en clínica obstétrica". Rev. Col. de Obst. y Gin. 1 (3) abril-mayo 1950.

3. DE LEE-GREENHILL.- "Choque obstétrico, síncope y muerte súbita". Princ. y Prac. de Obst. T. II 1.9.

4. MORAGUES B. J.- "Choque, colapso y síndrome poshemorrágico". Clin. Obst. cap. LII. 1948.

5. PEREZ MANUEL L.- "Choque obstétrico". Trat. Obst. II (p. 1013) 1945.

6. BOTELLA LL. J.- "Shock obstétrico". Pat. Obst. Lec. 34 (p. 493). 1950.

7. JIMENEZ DIAZ.- "El colapso y el shock". Lecciones de Pat. Med. II (Lec. LXXXIII). 1940.

8. BATTRO ANTONIO.- "Iratamiento de la insuficiencia circulatoria aguda”. Terap, Clin. Cardini. I (cap. XII). 1945.

9. STEAD Jr. E. A.- "Colapso circulatorio y shock". Trat. de Med. Int. Cecil. II (p. 1962) 1945.

10. SODEMAN W. A.- "Shock". Fisiopat. Clin. Cap. I. (p. 37) 1952.

11. SELYE HANS.- "The story o fthe Adaptation syndrome", 1952.

12. ARMSTRONG T. y POZO J. R.- "Reporte preliminar sobre el uso del ACT'H endovenoso, en el tratamiento del Shock", reimpreso de Arch. Med. de Cuba, por Sinopsis Med. Internal. 1 (8). Nov. 1952. 